\title{
Study of Induced Current Electrical Impedance Tomography Configuration on 2 Dimensional Rectangular Object
}

\author{
Dudi Darmawan \\ Instrumentation and Control Research Group, Faculty of Industrial Engineering, Institut Teknologi Bandung. \\ Jl. Ganesha 10, Bandung 40132, Indonesia \\ NDT Research Group, Engineering Physics Study Program, Telkom University \\ Jl. Telekomunikasi 1, Bandung 40257, Indonesia \\ Email :dudiddw@gmail.com \\ Deddy Kurniadi \& Janivita S Sudirham \\ Instrumentation and Control Research Group, Faculty of Industrial Engineering, Institut Teknologi Bandung. \\ Jl. Ganesha 10, Bandung 40132, Indonesia \\ Email :kurniadi@tf.itb.ac.id; Janivita@tf.itb.ac.id \\ Suyatman \\ Engineering Physics Research Group, Faculty of Industrial Engineering, Institut Teknologi Bandung. \\ Jl. Ganesha 10, Bandung 40132, Indonesia \\ Email:yatman@tf.itb.ac.id
}

\begin{abstract}
Sensitivity issue becomes a common problem on Electrical Impedance Tomography. One effort to overcome this issue is through inducing a magnetic field. It needs proper inducer coil configuration in order to produce a good reconstruction result. In this study, inducer coil configuration is obtained to 10 configurations of a rectangular object. Reconstruction results showed that the better results achieved through increasing of induction numbers and in 50 percent of shifting between each induction.

Keywords : Sensitivity, Induced current, coil configuration, rectangular object
\end{abstract}

\section{Introduction}

ICEIT method is one of the imaging methods that is semi contactless ${ }^{1,2,3}$. Providing currents accomplished by inducing a magnetic field that is not touching the object. With such a system, the use of dual function electrodes and the magnitude of the applied current limitations, as well as on the applied-current electrical impedance tomography (ACEIT), is avoided ${ }^{4}$.

Moreover, the technique of current injection also determines current density formed on objects and can affect the imaging results ${ }^{5}$. In ACEIT, current injecting on the surface of the object boundary still leaves the problem of insensitivity of changes in object conductivity away from the boundary. While the induction of magnetic field on ICEIT is made across the surface of the object through a certain coil configuration $6,7$.

So the ability to detect the change of conductivity (distinguishability) is enhanced through the giving of appropriate flow pattern ${ }^{8,9}$. So that one of the important issue is how to get the better coil configuration that improve the distinguishability. Improved induction system configuration has been done through the addition of induction ${ }^{4}$, the use of coil-shaped orthogonal ${ }^{10}$, the rectangular shaped coil ${ }^{11}$ as well as coil segments ${ }^{8}$. Increasing the number of induction increase the amount of measurement data ${ }^{4}$ but the optimal position of each induction has not been determined yet.

In this study, simulation ICEIT on imaging with two-dimensional rectangular object is performed 
through a variety of number and position of induction. Thus, this paper is organized as follows. Section II explains the various configurations induction system to be simulated. Section III explains the relationship of physical parameters in the object model through forward and inverse models determination. Section IV describes the results of simulation models developed and reconstructed by inverse models obtained. And finally, all the simulation results are concluded in Section V.

\section{Induction System Configuration}

Parameters induction system are coil shape and its dimension, the number of coil windings, distance of induction, magnitude and frequency of current excitation. While the configuration of induction system regarding the number and arrangement of the coils which is attached on the surface of the object. The shape and dimensions of the coils determine a fill factor ratio which is defined as equation (1).

$$
\text { fill }_{\text {factor }}=\frac{\left(\text { Diameter }_{\text {coil }}\right)^{2}}{\left(\text { Diameter }_{\text {object }}\right)^{2}} \ldots \ldots
$$

In this study, the shape and dimension of the coils are the same with object, so fill factor equal to 1. Fill factor value as it is the minimum value which the coil is still quite representative induces object surface. In addition to the fill factor, the distribution of magnetic potential induced also determines the induction results. Magnetic field induction depends on the magnitude of current given and the distance to the object surface. The magnitude of the potential magnetic field is defined by equation (2).

$$
\vec{A}=\int \frac{\mu_{o} i}{4 \pi} \frac{\overrightarrow{d l} \times \hat{r}}{r}
$$

where

$\vec{A}=$ vector of magnetical potential

$\mu_{0}=$ vacuum permeability

$i=$ exitation current

$\overrightarrow{d l}=$ vector of coil lenght elemen

$\mathrm{r}=$ distance to elemen
For rectangular shaped coil, the calculation of magnetic field is done by summing the potential of 4 rectangular sides .So that the magnitude of the magnetic potential is formulated as a numerically equation (3).

$$
\begin{gathered}
\vec{A}=\sum_{i=1}^{4} \frac{\mu_{o} i}{4 \pi} \int\left[\left(\frac{z d x-y d z}{r}\right) \hat{\imath}+\left(\frac{x d z-z d x}{r}\right) \hat{\jmath}\right. \\
\left.+\left(\frac{y d x-x d y}{r}\right) \hat{k}\right] \ldots \ldots \ldots(3)
\end{gathered}
$$

Configuration is obtained by putting the center of induction coil into a number of specific coordinates on the surface of the object. Surface of the coil is made parallel to the surface of the object so that the induced current is on the surface surrounded by electrodes. Inducing is performed by sliding along the surface of the coil within the same hypothetical to the surface of the object, as Figure 1.

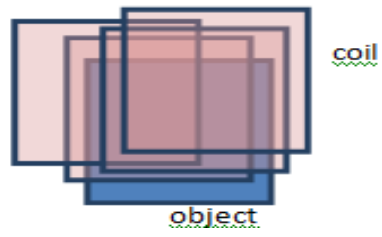

Fig. 1. Rectangular coil arrangement (red) on the top of object (blue)

Each configuration induction is distinguished by the number and coordinates of each induction. In this study, simulations of induction are conducted on ten kinds of configurations with any number of coordinate inductions as Figure 2.

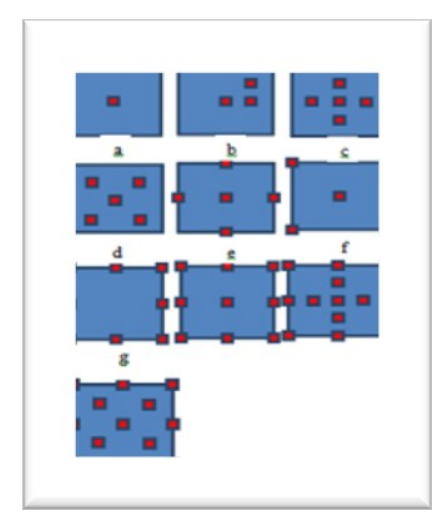

Fig. 2. 10 configuration of center point position of each coil (red) on the top of object (blue) are called (a) $1 \mathrm{~K}$ (b) $3 \mathrm{~K}$ (c) 
$5 \mathrm{KHV} 25$ (d) 5KDG_25 (e) 5KHV_50 (f) 5KDG_50 (g) $8 \mathrm{~K} 550$ (h) $9 \mathrm{~K} \_50$ (i) $13 \mathrm{KHV}$ (j) $13 \mathrm{KDG}$

\section{Forward and Inverse Model}

In ICEIT, the magnetic field is induced on the object through a current-carrying coil. Induced currents are formed in the object and generate potential distribution on the surface of the object. The physical relationship between the distribution of potential and magnetic potential based on Maxwell's equations. The formulation of relationship between conductivity, electrical potential, and induced magnetic field is generated as in equation (4).

$$
\nabla \cdot \sigma \nabla V=-\frac{d A}{d t} \nabla \sigma=-\omega A \nabla \sigma
$$

This equation is known as Poisson's equation.

ICEIT forward model is the Poisson equation solution which states the electric potential $\boldsymbol{V}$, on $\boldsymbol{\Omega}$ domain, as a function $\boldsymbol{F}$ of the electrical conductivity $\sigma$ and magnetic potential $\boldsymbol{A}$. The magnitude of magnetic potential depends on parameters such as the geometry of the magnetic induction coil $(\boldsymbol{C})$, excitation current $(\boldsymbol{I})$ and frequency of the excitation current $(\omega)$.

$$
\left.F(\underbrace{\sigma}_{\text {object parameter }}, \underbrace{C, I, \omega}_{\text {exitation coil parameter }}) \rightarrow V\right|_{\Omega}
$$

To make it easier to get the solution of the Poisson equation, a numerical approach is done using current conservation concept. This method is used through applying 2D integral formulation of the Poisson equation, in order to obtain equation (5).

$$
\iint_{\Omega_{S}} \nabla \times[\sigma \nabla V] d S=-\iint_{\Omega_{S}}(\omega A \nabla \sigma) d S
$$

If the divergence theorem is applied to equation (5) then equation (6) is obtained.

$$
\oint_{\Omega_{S}} \sigma \nabla V \cdot d l=-\iint_{\Omega_{S}}(\omega A \nabla \sigma) d S
$$

Numerical solution of the equation (7) produces equation (8).

$$
\sum_{l s e l} \sigma \nabla V \Delta l=-\omega A \nabla \sigma \Delta S
$$

The inverse model is generated by mapping the potential distribution to the conductivity distribution.

$$
\left.F^{-1}[\underbrace{\left.V\right|_{\partial \Omega_{l}}}_{\text {measurement }}, \underbrace{C, I, \omega}_{\text {exitation coil parameter }}] \rightarrow \sigma\right|_{\Omega}
$$

Through the use of linearization method then changes the conductivity becomes comparable to the potential boundary changes, according to equation (9).

$$
\Delta \bar{V}=S \Delta \bar{\sigma}
$$

$\boldsymbol{S}$ is the sensitivity matrix. Through the calculation of the inverse of sensitivity matrix, the change of conductivity distribution is obtained through a one-step solution, according to equation (9).

$$
\Delta \bar{\sigma}=S^{-1} \Delta \bar{V}
$$

So that absolute conductivity is

$$
\sigma_{\text {anomaly }}=\sigma_{\text {homogen }}+\Delta \bar{\sigma}
$$

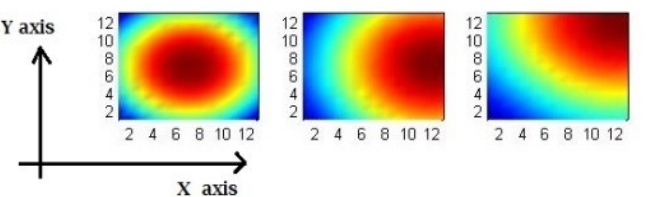

(a)
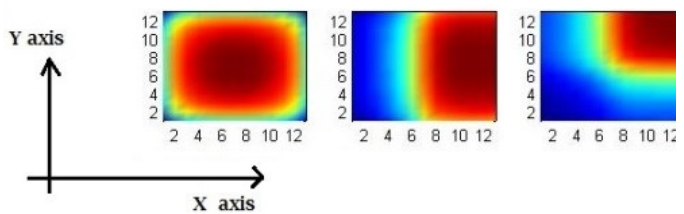

(b)
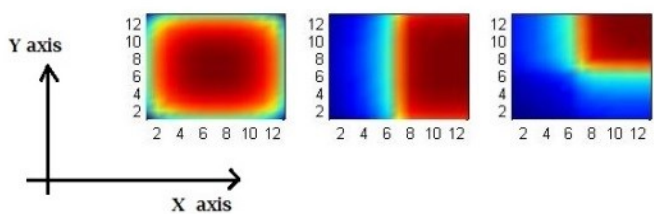

(c)

Fig. 3. Magnetic field distribution, in $X Y$ plane, from highest (red) $10^{-7}$ Tesla to lowest value (blue) $10^{-8}$ Tesla with the distances between coil and obiect 


\section{Simulation Results}

The first simulation is the determination of the distribution of the magnetic field using equation (3). Simulationis performed usingthe induction system parameters as follows:

The number of observation points $=144$

The number of coil windings $=40$

Diameter wire coil $=0.0001 \mathrm{~m}$

Maximum flow $=0.02 \mathrm{~A}$ with $\mathrm{f}=10 \mathrm{kHz}$

Simulation results are shown in Figure 3.

Figure 3 shows the distribution of magnetic field induction occured at 3 points in the object, namely a shift $50 \%$ to the right, $50 \%$ to the right and upward, and $50 \%$ to the upward. The simulation results show that the closer the distance of induction the better homogeneous of magnetic field distribution.

The distribution of the magnetic field that is given as a function of time induce current in the object. Thus, the potential distribution on the surface of the object is calculated using equation (7). The simulation result of determining the potential distribution is shown in Figure 4.

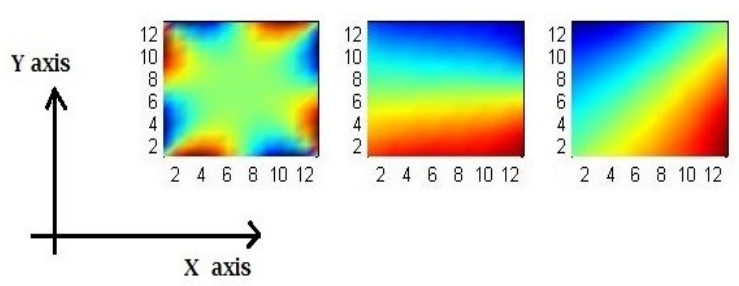

Fig. 4. The distribution of electric potential, in XY plane, from highest (red) to lowest value (blue) with 3 inductions.

Furthermore, the simulation is done to obtain the results of reconstruction with some configurations induction system. Simulations performed using the inverse model of the 3 anomalous resistivity distributions, such as Figure 5, where homogen resistivity is $1000 \mathrm{Ohm} / \mathrm{m}^{2}$ (blue) and anomalous resistivity is $2000 \mathrm{Ohm} / \mathrm{m}^{2}$ (red). This anomalous configuration can be realized experimentally such as a beam in the soil.

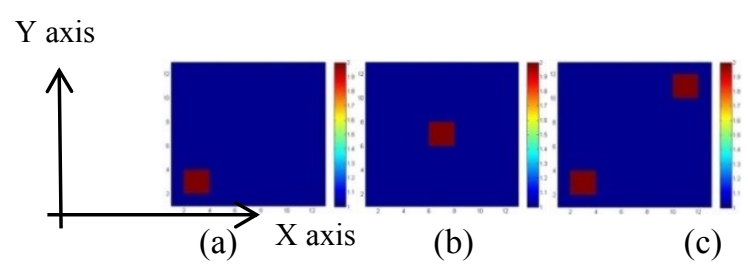

Fig. 5. Anomalous resistivity distribution types, in XY plane, (a) side (b) center (c) twin

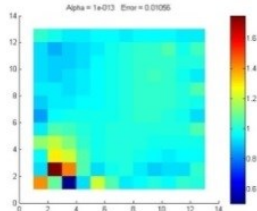

(a)

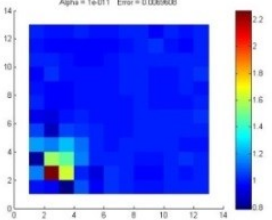

(c)

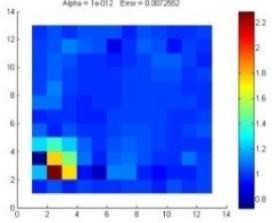

(e)

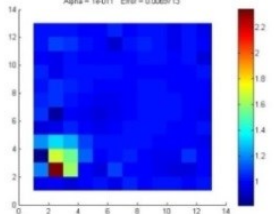

$(\mathrm{g})$

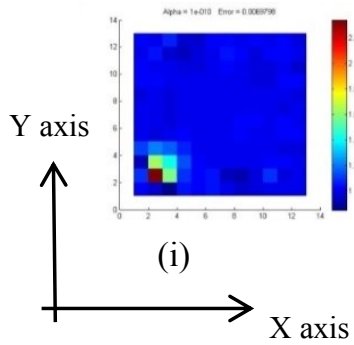

Fig. 6. Reconstruction results of side anomaly , in XY plane, for (a) model $1 \mathrm{~K} \quad$ (b) model $3 \mathrm{~K}$ (c) model 5KHV_25 (d) model 5KDG_25 (e) model 5KHV_50 (f) model 5KDG 50 (g) model 8K_50 (h) model 9K_50 (i) model $13 \mathrm{KHV}$ (j) model $13 \mathrm{KDG}$

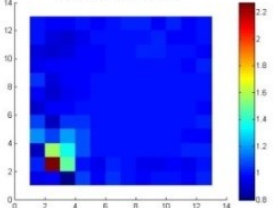

(b)

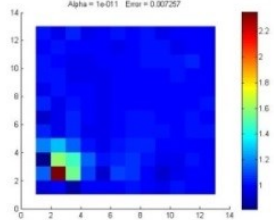

(d)

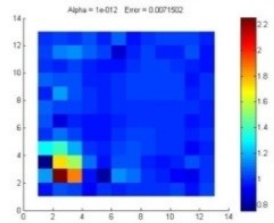

(f)

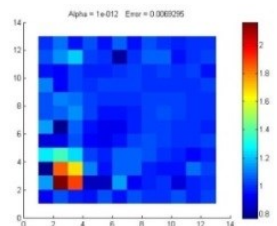

(h)

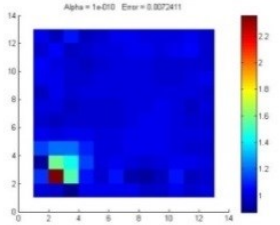

(j) 
To get resistivity distribution from the boundary potential distribution observed is called reconstruction process. Reconstruction is conducted by induction distance of $10 \mathrm{~cm}$, the maximum induced current of 20 $\mathrm{mA}$, and $10 \mathrm{KHz}$ of excitation current frequency. Simulations were performed for three types of anomalous in 9 inductions. The reconstruction results are such as Figures 6, 7, and 8, where anomalous in red and homogen resistivity in blue.

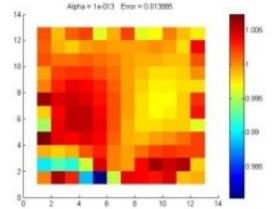

(a)

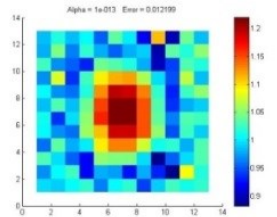

(c)

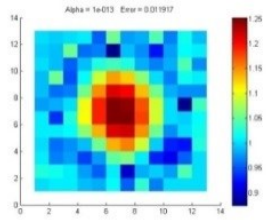

(e)

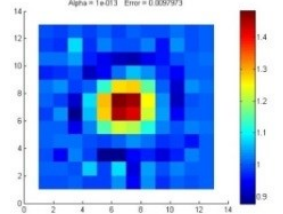

(g)

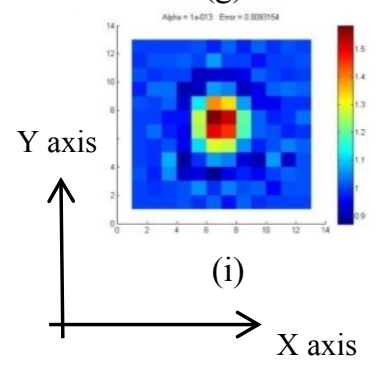

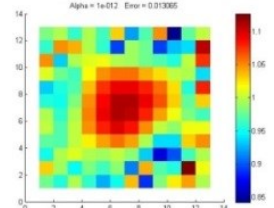

(b)

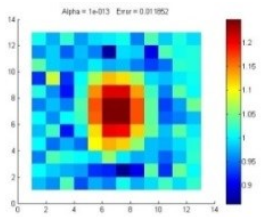

(d)

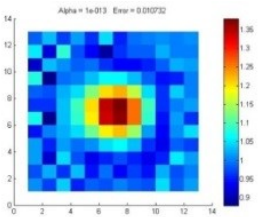

(f)

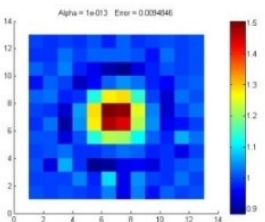

(h)

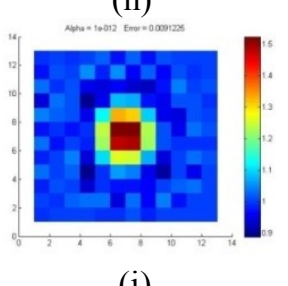

(j)
Figure 7. Reconstruction results of center anomaly, in $\mathrm{XY}$ plane, for (a) model $1 \mathrm{~K}$ (b) model $3 \mathrm{~K}$ (c) model 5KHV_25 (d) model $5 \mathrm{KDG} \_25$ (e) model $5 \mathrm{KHV} 50$ (f) model $5 \mathrm{KDG} 550$ (g) model $8 \mathrm{~K} \_50$ (h) model $9 \overline{\mathrm{K}} \_50$ (i) model $13 \mathrm{KHV}$ (j) model $13 \mathrm{KDG}$

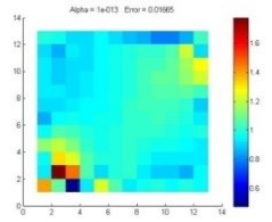

(a)

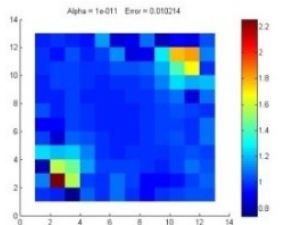

(c)

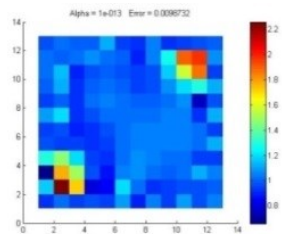

(e)

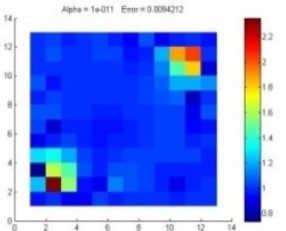

(g)

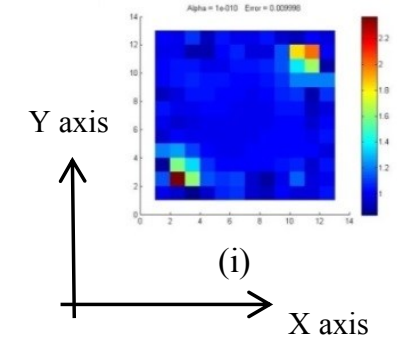

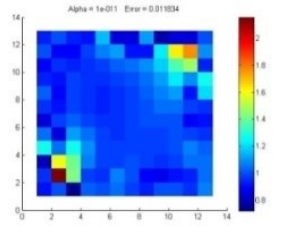

(b)

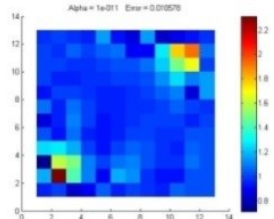

(d)

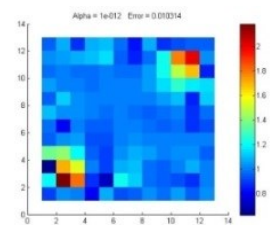

(f)

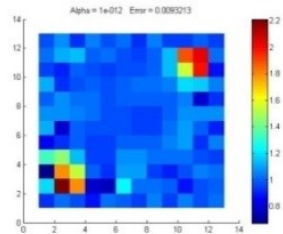

(h)

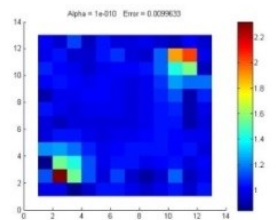

(j)
Fig. 8. Reconstruction results of twin anomaly, in $X Y$ plane, for (a) model $1 \mathrm{~K}$ (b) model $3 \mathrm{~K}$ (c) model 5KHV_25 (d) model 5KDG_25 (e) model 5KHV_50 (f) model 5KDG_50 (g) model 8K_50 (h) model 9K_50 (i) model 13KHV (j) model $13 \mathrm{KDG}$

Simulation results, as shown in Figure 6-8, visually demonstrate that the detection of the anomaly on the edge (side and twin) generally do not show a significant difference in the results if the number of induction is increased. As for the anomalous in the middle (center), the result of reconstruction is getting better as the increase of the induction number. The reconstruction results are evaluated numerically using the parameter of 
root mean square (rms) of the difference in the test resistivity distribution with the distribution of resistivity reconstruction results, such as equation (11).

$$
\text { Error }=\frac{1}{N} \sqrt{\left(\rho_{\text {reconstruction }}-\rho_{\text {test }}\right)^{2}}
$$

Summary of simulation results with error values of each are shown in Table 1.

The results of error calculations showed that an increasing number of inductions give the average error of the third anomaly becomes smaller. On the other hand, an addition of induction number increase the computational complexity of the measurement and the longer.

Table 1. Summary of simulation results error.

\begin{tabular}{|c|c|c|c|c|}
\hline Model & $\begin{array}{c}\text { Error } \\
\text { Side }\end{array}$ & $\begin{array}{c}\text { Error } \\
\text { Center }\end{array}$ & $\begin{array}{c}\text { Error } \\
\text { Twin }\end{array}$ & $\begin{array}{c}\text { Average } \\
\text { Error }\end{array}$ \\
\hline 1K & 0,0106 & 0,01389 & 0,01665 & 0,01370 \\
\hline 3K_25 & 0,0077 & 0,01307 & 0,01183 & 0,01087 \\
\hline 5KHV_25 & 0,0070 & 0,01220 & 0,01021 & 0,00979 \\
\hline 5KDG_25 & 0,0073 & 0,01185 & 0,01058 & 0,00990 \\
\hline 5KHV_50 & 0,0073 & 0,01192 & 0,00987 & 0,00968 \\
\hline 5KDG_50 & 0,0072 & 0,01073 & 0,01031 & 0,00940 \\
\hline 8K_50 & 0,0070 & 0,00980 & 0,00942 & 0,00870 \\
\hline 9K_50 & 0,0069 & 0,00949 & 0,00932 & 0,00858 \\
\hline 13KHV & 0,0070 & 0,00932 & 0,00999 & 0,00876 \\
\hline 13KDG & 0,0072 & 0,00912 & 0,00996 & 0,00878 \\
\hline
\end{tabular}

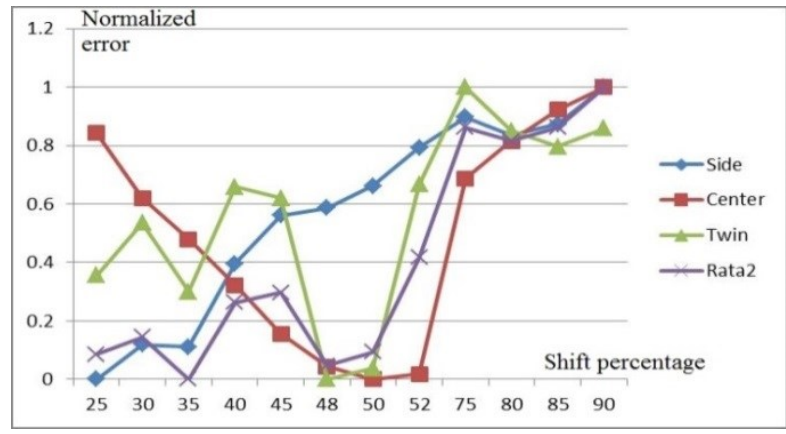

Fig. 9. Error with shifting on 5 times induction

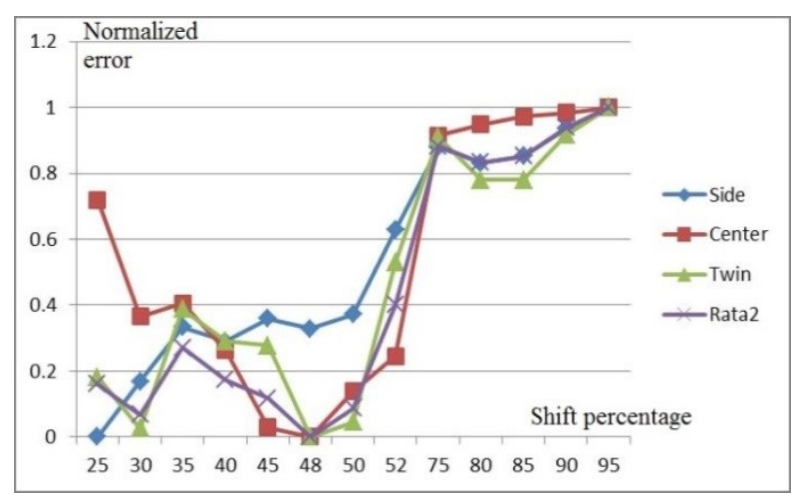

Fig. 10. Error with shifting on 9 times induction

Variation of induction positions are performed to know an influence of induction position shifts. Shifting of induction position is addressed through the change of distance between each induction point. The magnitude of shifting is expressed in percentage form toward the wide of the object side. Error of reconstruction for some shift values on 5 and 9 times inductions are summarized in Figure 9 and 10.

The results of simulation show that the smallest average error of the third anomalyis achieved on some particular value. The shift which is made smaller or larger than half the length of the coil side made the error to be bigger. The simulation results indicate that the position of induction affects the reconstruction results and there is a shift in the optimal value.

The next simulation is conducted to determine the effect of induction distance on the result of the reconstruction. Simulations are performed with induction distance of $\mathrm{z}=50 \mathrm{~cm}, \mathrm{z}=40 \mathrm{~cm}, \mathrm{z}=30 \mathrm{~cm}, \mathrm{z}$ $=20 \mathrm{~cm}, \mathrm{z}=10 \mathrm{~cm}$, and $\mathrm{z}=1 \mathrm{~cm}$. Reconstruction results are shown in Table 2.

Table 2. Error for any distance

\begin{tabular}{|c|c|c|c|}
\hline \multirow{2}{*}{$\begin{array}{c}\text { Distance } \\
(\mathbf{c m})\end{array}$} & $\begin{array}{c}|c| \\
\text { Anomaly } \\
\text { Side }\end{array}$ & $\begin{array}{c}\text { Anomaly } \\
\text { Center }\end{array}$ & $\begin{array}{c}\text { Anomaly } \\
\text { Twin }\end{array}$ \\
\cline { 2 - 4 } & 0.00887 & 0.01166 & 0.01296 \\
\hline 1 & 0,00949 & 0,01298 & 0,01383 \\
\hline 10 & 0,00975 & 0,01315 & 0,01398 \\
\hline
\end{tabular}




\begin{tabular}{|c|c|c|c|}
\hline 30 & 0,00988 & 0,01331 & 0,01398 \\
\hline 40 & 0,00994 & 0,01342 & 0,01397 \\
\hline 50 & 0,00995 & 0,01348 & 0,01398 \\
\hline
\end{tabular}

Simulation results showed that generally the greater the distance the greater the error of reconstruction. It shows that the reconstruction should be better if is performed as close as possible to the surface of the object. These results are associated with the magnetic potential distribution, as Figure 6. The closer the further induction produces a homogeneous distribution of magnetic potential.

\section{CONCLUSIONS}

Configuration induction produces better reconstruction results if the number of induction increased. But an increasing of induction number is coupled with the increasing complexity of the measurement and the longer computational time.

Shifting between each induction coil affect the reconstruction result obtained. Detection of an anomaly on the object produced good results through specific configuration of induction system with particular induction position.

Distance of induction determines the distribution of the magnetic field that induces the object. The closer the induction made more homogeneous distribution of the magnetic field induced in the region. Homogeneity of the magnetic field distribution determines the outcome of reconstruction performed.

\section{Reference}

1. Purvis, W. R.; R. C. Tozer; and I. L. Freeston, Impedance imaging using induced currents, in Proc. 12th annu. Int. Conf. IEEE Eng. Med. Biol. Soc., vol. 12, pp. 114-115, 1990.

2. Purvis, W. R.; R. C. Tozzer; D. K. Anderson; I. L. Freeston, Induced current impedance imaging, IEEE. Proc., pt. A, vol. 140, no. 2, pp. 135-141, Mar. 1993.

3. Zlochiver, Sharon; M Michal Radai; Shimon Abboud; Moshe Rosenfeld; Xiu-Zhen Dong; Rui-Gang Liu; FuSheng You; Hai-Yan Xiang and Xue-Tao Shi, Induced current electrical impedance tomography system: experimental results and numerical simulations, IOP Publishing Ltd, 2004.
4. Gencer, Nevzat, G.; Y. Ziya Ider, Electrical Impedance Tomography : Induced-Current Imaging Achieved with a Multiple Coil System, IEEE Transactions on Biomedical Engineering, vol. 43, No. 21996.

5. Adler, Andy; P. O. Gaggero; and Y. Maimaitijiang, Adjacent simulation and measurement patterns considered harmful, Physiological Measurement, 2011.

6. Gencer, N. G.; Y. Z. Ider, A Comparative study of several exiciting fields for induced current EIT, Physiol. Meas., vol. 15, pp. A51-A57, 1994.

7. Gursoy, Doga; Yasin Mamatjan; Andy Adler; and Hermann Scharfetter, Enhancing impedance imaging through multimodal tomography, 2012.

8. Eyüboğlu, B. M.; A. Köksal; and M. Demirbilek. Distinguishability analysis of an induced current EIT system using discrete coils. Phys. Med. Biol. 45:1997-2009, 2000.

9. Isaacson, D., Distinguishability of conductive by electric current tomography, IEEE Trans. Med. Imag., vol 5, pp 91 95, 1986.

10. Grimberg, R.; Savin, A.; Radu, E.; Mihalache, O., "Nondstructive Evaluation of The Severity of Diskontinuities in Flat Conductive Materials by an Eddy Current Transducer with Orthogonal coils", IEEE Trans. Magn, 2000.

11. Yunze, He; Pan, Mengchun; Luo, Feilu; Tian, Guiyun, "Pulsed Eddy Current Imaging and Frequency Spectrum Analysis for Hidden Defect Nondestruvtive Testing and Evaluation", NDT\&E International, 2011. 